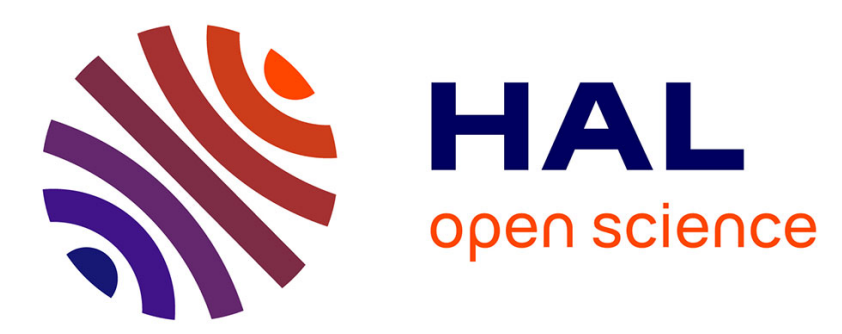

\title{
Fast Reconstitution Method (FRM) to Compute the Broadband Spectrum of Common Mode Conducted Disturbances
}

\author{
Denis Labrousse, Bertrand Revol, Cyrille Gautier, Francois Costa
}

\section{To cite this version:}

Denis Labrousse, Bertrand Revol, Cyrille Gautier, Francois Costa. Fast Reconstitution Method (FRM) to Compute the Broadband Spectrum of Common Mode Conducted Disturbances. IEEE Transactions on Electromagnetic Compatibility, 2013, 55 (2), pp.248 - 256. 10.1109/TEMC.2012.2219056 . hal01735861

\section{HAL Id: hal-01735861 \\ https://hal.science/hal-01735861}

Submitted on 21 Mar 2018

HAL is a multi-disciplinary open access archive for the deposit and dissemination of scientific research documents, whether they are published or not. The documents may come from teaching and research institutions in France or abroad, or from public or private research centers.
L'archive ouverte pluridisciplinaire HAL, est destinée au dépôt et à la diffusion de documents scientifiques de niveau recherche, publiés ou non, émanant des établissements d'enseignement et de recherche français ou étrangers, des laboratoires publics ou privés. 


\title{
Fast Reconstitution Method (FRM) to Compute the Broadband Spectrum of Common Mode Conducted Disturbances
}

\author{
Denis Labrousse, Bertrand Revol, Member, IEEE, Cyrille Gauthier, Member, IEEE, François Costa, \\ Member, IEEE
}

\begin{abstract}
This paper deals with time domain simulation of common mode (CM) conducted disturbances in power electronics. The study proposes a proof of concept of a Fast Reconstruction Method (FRM) which allows to compute the spectrum of a common mode current based on the knowledge of a single pulse of current (i.e. the knowledge of the disturbances on a small fraction of time) and of the switching instants (i.e. the control strategy of the structure). The first parts of the paper demonstrate the reliability of the method using data measurements of a common mode current generated by a PWM half bridge converter and then by a three phase inverter. The simulation time saved thanks to the FRM is then quantified by comparing the simulation durations with or without using the FRM. In our example, the FRM allows to divide the simulation time by about 10 .

This analysis points out that the complex information of a $\mathrm{CM}$ current is contained in a small time interval. As a consequence the complete spectrum can be predicted with only a very fast simulation. The mathematical techniques developed here forecast promising reduction of simulation durations.
\end{abstract}

Index Terms - emc, emi, common mode, power electronics, circuit simulation, computation times.

\section{INTRODUCTION}

$\mathrm{N}$ UMERICAL simulations have become, like in many other domains one of the major tools available to study and analyze phenomena in power electronics electromagnetic compatibility. Circuit simulation (Pspice, Simplorer...) in particular is among the most commonly used type of simulation [1], [2]. They allow to easily estimate the conducted disturbances generated by a power electronic structure although on a limited frequency band. Indeed, above a few $\mathrm{MHz}$, results often lack precision. This approach remains perfectly adapted to design converters since only functional aspects of the structure need then to be taken into account. However, when one aims to precisely evaluate disturbances, the usual models need to be more detailed.

The authors are with the Systèmes et Applications des Technologies de l'Information et de l'Energie (SATIE ENS de Cachan) Laboratory, Cachan 94230, France ((e-mail: denis.labrousse@satie.ens-cachan.fr; bertrand.revol@satie.ens-cachan.fr; francois.costa@satie.ens-cachan.fr)
Electrical components in particular have to be described with all their stray elements including the ones due to the integration in the complex electromagnetic environment [3-5]. This increasing complexity of models unfortunately directly impacts on the time of simulation that can become unreasonably long. Equations to solve become at the same time more complex which may lead to numerical instability and solver convergence problems [6], [7]. The enormous recent improvements of calculation means are not yet sufficient to allow a precise simulation of a complex structure on a large frequency band. However, that kind of tool could greatly benefit manufacturers. Indeed, in standard design flows, EMC design is tested only after a prototype has been achieved. Manufacturers aim to integrate EMC design earlier in the conception, ideally during the virtual prototyping stage, in order to reduce the design time and thus the design costs (Fig. 1). Virtual prototyping requires precise and fast tools capable of estimating conducted and radiated disturbances. In this paper, we propose a new method which could reduce duration of time domain simulations. This study highlights that it is not always necessary to perform the simulation on a wide time domain to obtain the global perturbation spectrum on the whole frequency range. We will focus here on common mode $(\mathrm{CM})$ perturbations and detail how the time interval necessary for an accurate simulation can be largely reduced. This paper is organized as follows. Section II focuses on a mathematical description of a common mode current and introduces the developed method. Section III deals with the treatment of data acquisition of a common mode current generated by a PWM half bridge converter. Its aim is to prove that, on a real common mode current, a small part of the data contains all the information required to compute the spectrum on a large frequency band. This study, which constitute of proof of concept, was voluntarily carried out with measurements and not simulations. This ensures that the method does not require any hypothesis but can be applied to real structures. All the experiments have been performed on the largest possible frequency range which was imposed by the sampling rate. Each time, the frequency range is larger than the EMC conducted emission standard band $(150 \mathrm{kHz}-$ $30 \mathrm{MHz}$ ). In section IV, the same methodology is applied to a more complex structure: a three-phase inverter feeding a synchronous machine. Finally, in section V, the method is 
applied to a time domain simulation in order to quantify the reduction of simulation duration.

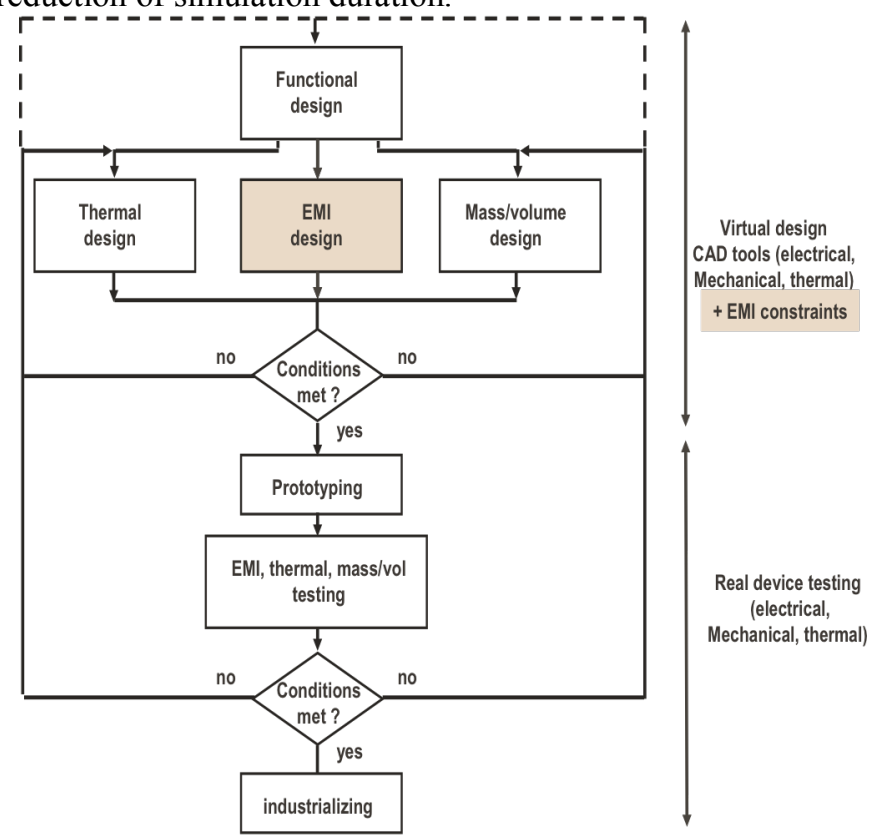

Fig. 1: Industrial design flow highlighting the need to integrate EMC constraint as soon as possible.

Tip to the reader

The electrical quantities are expressed in time domain with small characters and in frequency domain with capital letters. For example, $x$ in the time domain becomes $X$ in the frequency

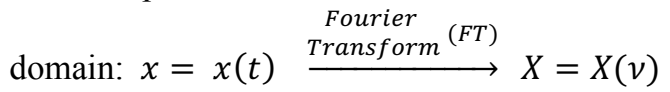

\section{COMmon Mode Current Analysis}

A typical common mode current $\left(i_{C M}\right)$ can be considered as a succession of different pulses $\left(p_{k}\right)$ which appear at welldefined times $\left(t_{k}\right)$ imposed by the control strategy of the structure. Its shape is imposed by the propagation path followed by the $\mathrm{CM}$ current and by the equivalent $\mathrm{CM}$ voltage source which generates it [8-10]. Its expression in the time domain is given by (1) and in the frequency domain by (2).

$i_{C M}=\sum_{k=1}^{n}\left[p_{k} * \delta\left(t-t_{k}\right)\right]$

with $n$ the number of pulses

$$
\text { and } \quad \begin{aligned}
& \delta(t)=0, \forall t \neq 0 \\
& \delta(0)=1
\end{aligned}
$$

$I_{C M}=\sum_{k=1}^{n} P_{k} \cdot e^{-2 \pi \cdot j \cdot v \cdot t_{k}}$

In order to calculate the spectrum of any periodic signal, it is necessary to have the knowledge of its waveform in the time domain over one period. In power electronics structures, the period of signals (in particular the CM current period) depends on the control strategy. Two different cases can be considered.
The first case concerns structures with a constant duty cycle (typically DC-DC converters). In such structures and in the steady state, the duration of the period is equal to the switching period. Simulations thus do not require long computing time. In the second case the duty cycle is not constant overtime (DC-AC converters). In that case, the minimum simulation duration needed to compute the whole perturbation spectrum is equal to the lower frequency period. For example, to calculate the spectrum of a $\mathrm{CM}$ current generated by an inverter supplying a motor, the temporal window to consider is equal to the period of the power currents. It can be quite important (typically several tens of $\mathrm{ms}$ ) and can thus generate substantial computing time.

The study that follows shows that it is possible to determine a single pulse $(\hat{P})$ that will enable to calculate the entire spectrum using (3) with the knowledge of all the switching instants $\left(t_{k}\right)$. Such pulse can be chosen among the measured ones (4). We believe that this pulse can also be obtained thanks to a short and precise time domain simulation for a specific operating point. The synoptic of the proposed method named FRM (Fast Reconstitution Method) is represented in Fig. 2. In the next sections, a pulse $(\widehat{P})$ will be calculated from data measurement in order to demonstrate what the FRM is reliable. Experimental data will also be used to explain how a significant pulse can be extracted from a CM current.

$$
\begin{aligned}
& I_{C M} \approx \hat{P} \cdot \sum_{k=1}^{n} e^{-2 \pi \cdot j \cdot v \cdot t_{k}} \\
& \hat{I}_{C M}=P_{m} \cdot \sum_{k=1}^{n} e^{-2 \pi \cdot j \cdot v \cdot t_{k}}, m \in[1, n]
\end{aligned}
$$

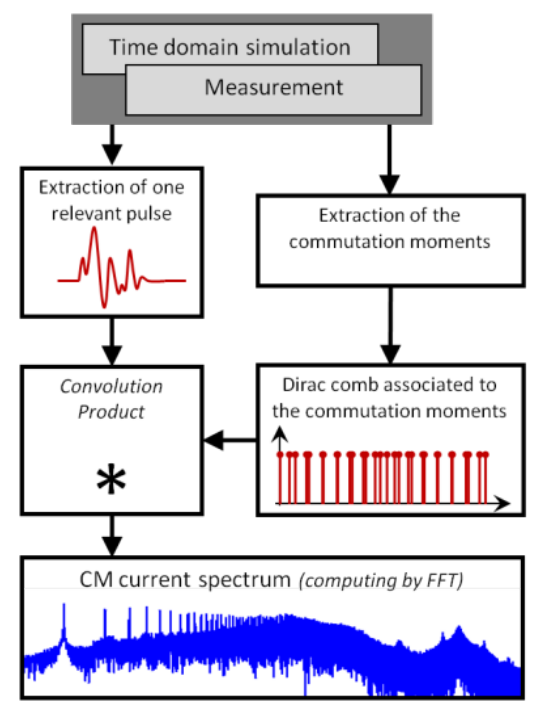

Fig. 2: Synoptic of the FRM (Fast Reconstruction Method).

\section{Application to a PWM Half Bridge Converter}

This part deals with the analysis of the CM current generate by a PWM half bridge converter.

\section{A. Experimental test bench}

The experimental test bench used is made of a switching cell connected to a $[R, L]$ load (Fig. 3). From the CM point of 
view, the DC power supply is insulated from the converter thanks to a CM filter. The DC bus voltage is equal to $50 \mathrm{~V}$. The control strategy used is a pulse width modulation (PWM) strategy. The switching frequency is equal to $10 \mathrm{kHz}$ and the modulation signal is equal to $400 \mathrm{~Hz}$ (Fig. 4). This imposes a variable load current $\left(i_{L}(t)\right)$ centered on about $4 \mathrm{~A}$ with an amplitude of 4 A (Fig. 5). Fig. 6 shows the measurement of the CM current during two low frequency (LF) periods. An amplitude modulation of the CM current appears over time. This modulation is due to the fact that the semiconductors behavior depends on the load current intensity. As a consequence an evolution of the transient of the $\mathrm{CM}$ voltage $\left(d V_{C M} / d t\right)$ appears which in turn modulates the CM current. Consequently, the CM current contains an LF harmonic.

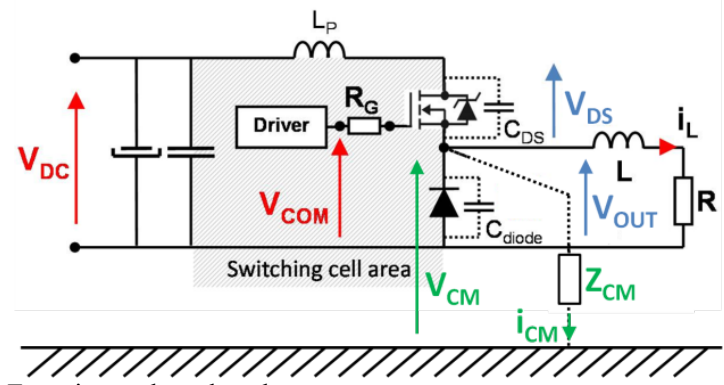

Fig. 3: Experimental test bench.

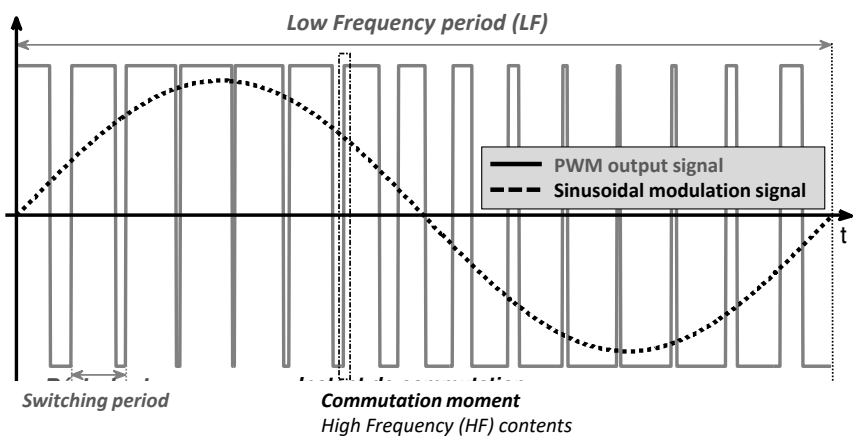

Fig. 4: Representation of different time intervals.

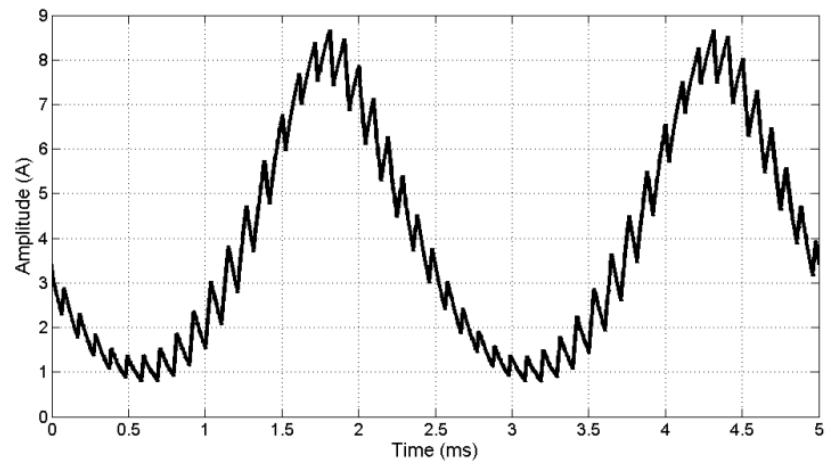

Fig. 5: Temporal waveform of the current load (iL).

\section{B. CM current decomposition as a function of the sign of $d V_{C M} / d t$}

The CM current pulses can be separated into two categories depending on whether the diode or the transistor imposes the switching. The pulses $p_{U_{k}}$ and $p_{D_{k}}$, correspond respectively to the case $d V_{C M} / d t>0$, when the diode imposes the switching and to the case $d V_{C M} / d t<0$, when the transistor imposes the switching. The switching instants $t_{U_{k}}$ and $t_{D_{k}}$ associated to the pulses $p_{U_{k}}$ and $p_{D_{k}}$ enable to define two partial currents $i_{C M-U}$ and $i_{C M-D}(5)$ which are presented on Fig. 7.

$$
\begin{aligned}
i_{C M-U} & =\sum_{k=1}^{N_{U}}\left[p_{U_{k}} * \delta\left(t-t_{U_{k}}\right)\right] \\
i_{C M-D} & =\sum_{k=1}^{N_{D}}\left[p_{D_{k}} * \delta\left(t-t_{D_{k}}\right)\right]
\end{aligned}
$$

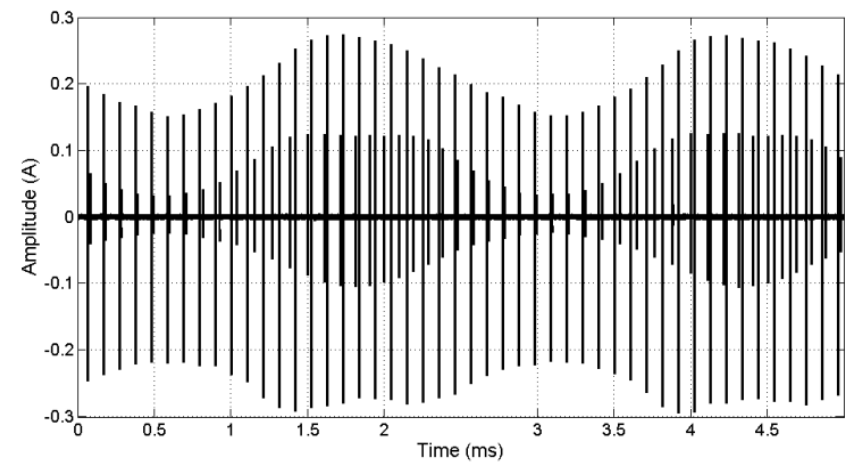

Fig. 6: Temporal waveform of the CM current (i $\mathrm{i}_{\mathrm{CM}}$ ).
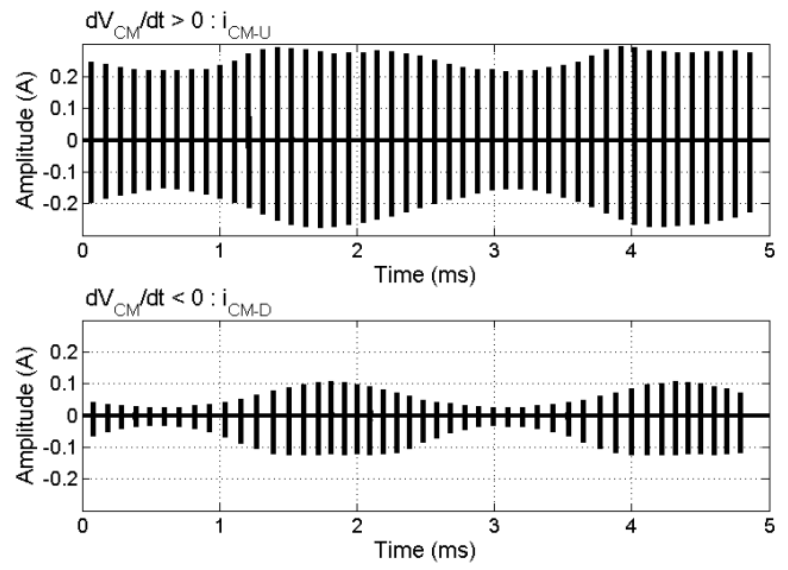

Fig. 7: Decomposition of the CM current (icM) into two partial currents depending on the sign of $d V_{C M} / d t$.

\section{Calculation of two equivalent pulses}

Two equivalent signals $i_{C M-U}^{\left(*^{-1}\right)}$ and $i_{C M-D}^{\left(*^{-1}\right)}$ can be obtained by calculating the deconvolution $\left(*^{-1}\right)$ of the partial CM currents with the Dirac comb associated to the switching instants (6).

$$
\begin{aligned}
& \left.i_{C M-U}^{\left(*^{-1}\right)}=i_{C M-U}\left(*^{-1}\right) \sum_{\substack{k=1 \\
N_{D}}}^{N_{U}} \delta\left(t-t_{U_{k}}\right)\right] \\
& i_{C M-D}^{\left(*^{-1}\right)}=i_{C M-D}\left(*^{-1}\right) \sum_{k=1}^{N_{D}}\left[\delta\left(t-t_{D_{k}}\right)\right]
\end{aligned}
$$

where $\left(*^{-1}\right)$ is the operation of deconvolution

These two calculated signals are represented on Fig. 8. Both signals evolve over the whole duration of the LF period. However, it can be noticed that the most important variations are limited in time. Indeed, the shape of the signals on the first 
switching period is very close to any $\mathrm{CM}$ current pulse while the rest of the signal is almost equal to zero. This enables to extract two equivalent pulses $\widehat{p_{U}}$ and $\widehat{p_{D}}$ defined on an HF period. These two pulses are represented in Fig. 9.
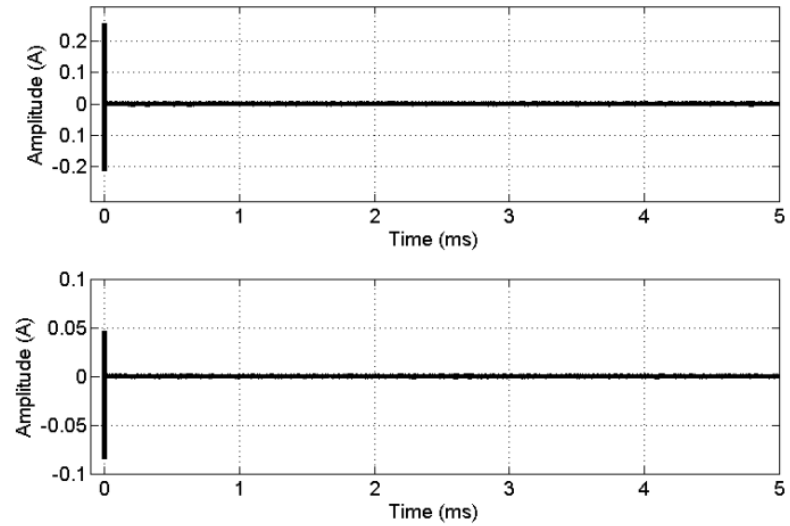

Fig. 8: Equivalent signals $i_{C M-U}^{\left(*^{-1}\right)}$ and $i_{C M-D}^{\left(*^{-1}\right)}$ obtained by deconvolution of $i_{C M-}$ $U$ and $i_{C M-D}$ by the switching instants.
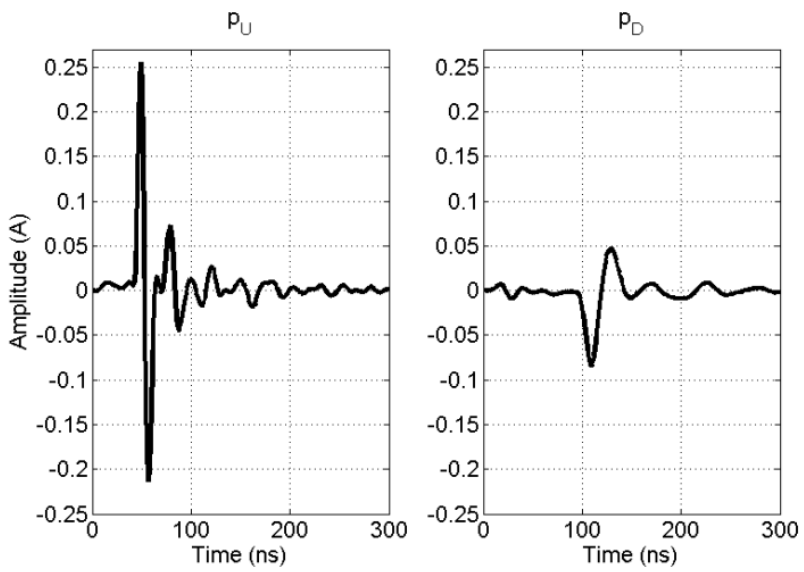

Fig. 9: Equivalent pulses extracted from $i_{C M-U}^{\left(*^{-1}\right)}$ and $i_{C M-D}^{\left(*^{-1}\right)}$.

An estimated $\mathrm{CM}$ current $\left(\widehat{l_{C M}}\right)$ can thus be computed using equation (7). This calculated current is represented in the time domain in Fig. 10 and the comparison in the frequency domain with the measured current is represented in Fig. 11. In the time domain, the LF modulation of the amplitude is lost since the $\mathrm{CM}$ current is made of only two pulses repeated in time with the same amplitude. However, the reconstruction in the frequency domain is very close to the initial spectrum from 10 $\mathrm{kHz}$ to $100 \mathrm{MHz}$.

$$
\widehat{l_{C M}}=\widehat{p_{U}} * \sum_{k=1}^{N_{U}} \delta\left(t-t_{U_{k}}\right)+\widehat{p_{D}} * \sum_{k=1}^{N_{D}} \delta\left(t-t_{D_{k}}\right)
$$

This first step shows that the knowledge of the switching instants and of two pulses is sufficient to represent the CM current in the frequency domain from the switching frequency to $100 \mathrm{MHz}$. The next part highlights the fact that it is also possible to extract from the measurement two pulses as relevant as the equivalent ones.
D. Selection from the measurement of the most relevant pulses

1) Selection using the maximum of the correlation coefficient between measured and equivalent pulses

In this section, we aim to demonstrate that, among all the pulses (Fig. 12) which constitute a CM current, it is possible to find two pulses as relevant as the equivalent ones. Each of the measured pulses $p_{U_{k}}$ (respectively $p_{D_{k}}$ ) is tested by computing the value of its correlation coefficient $C\left[p_{U_{k}} ; \widehat{p_{U}}\right]$ (respectively $C\left[p_{D_{k}} ; \widehat{p_{D}}\right]$ ) with the previously computed equivalent pulse $\widehat{p_{U}}$ (respectively $\widehat{p_{D}}$ ).

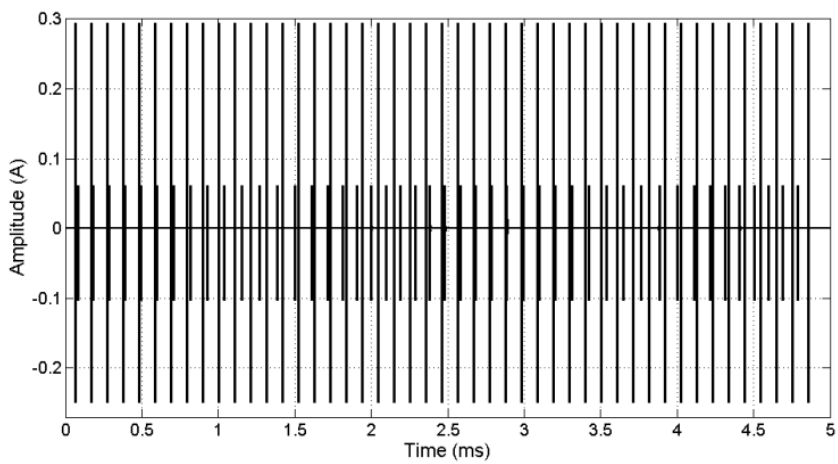

Fig. 10: Temporal waveform of the estimated CM current $\widehat{C M}$.

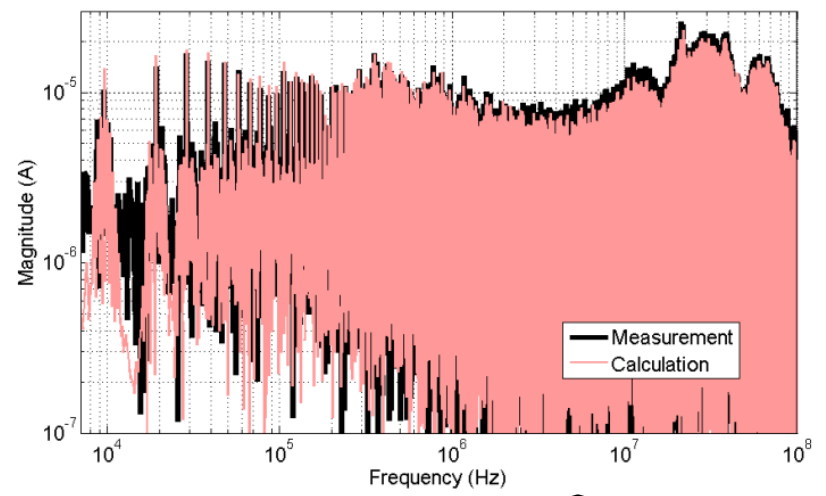

Fig. 11: Measured $\left(I_{C M}\right)$ and estimated CM current $\left(\widehat{I_{C M}}\right)$.
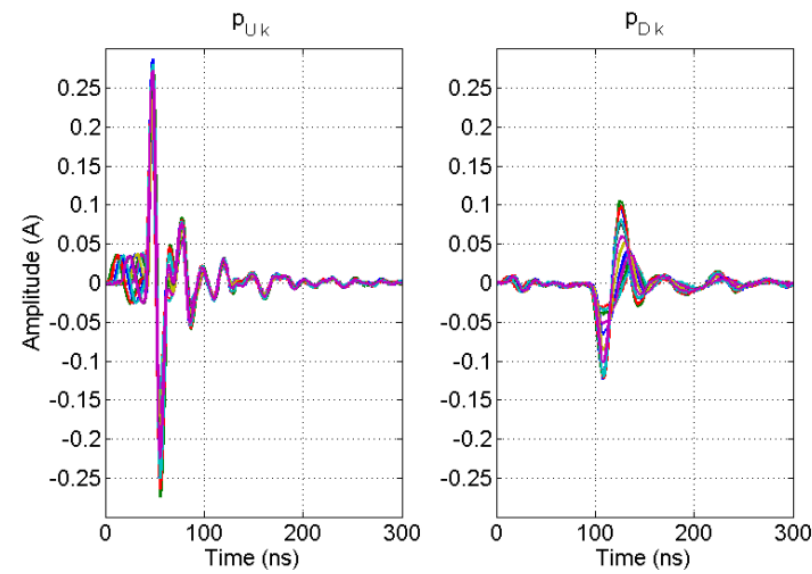

Fig. 12: Representation of some of the pulses which compose $i_{C M-U}$ and $i_{C M-D .}$. 
The representation of the correlation coefficient as a function of the pulse index is given by Fig. 13-A. It appears that all $p_{U-k}$ pulses correlate very well with $\widehat{p_{U}}$. On the contrary, the correlation coefficient $C\left[p_{D_{k}} ; \widehat{p_{D}}\right]$ varies between 0.69 and 0.99 with a periodic motive corresponding to the variation of the magnitude of the switched current (Fig. 13-B).
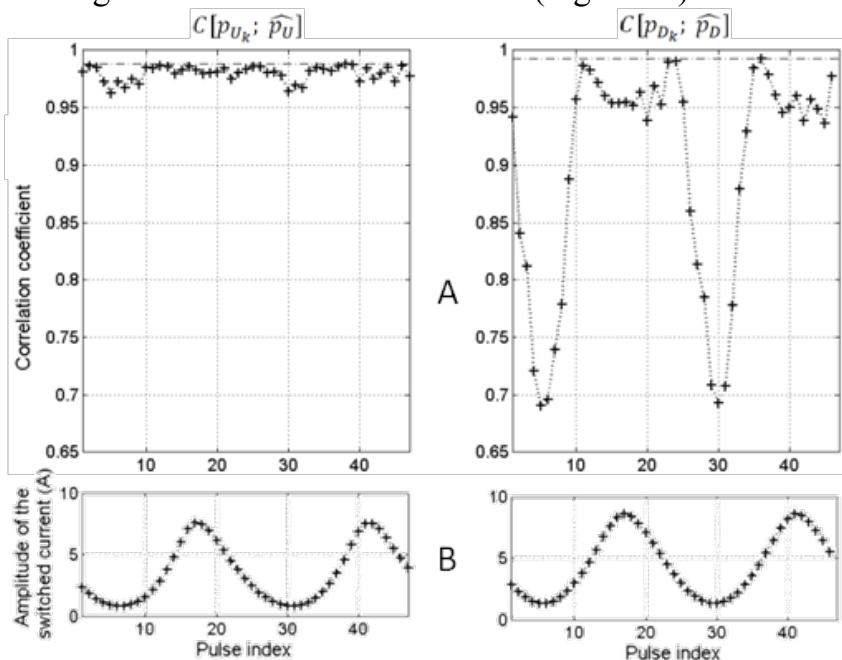

Fig. 13: A - Representation of the correlation coefficient calculated between the equivalent pulses $\widehat{p_{U}}$ and $\widehat{p_{D}}$ and all the extracted pulses. B - Magnitude of the switched current versus pulse index.

In order to explain the values of the correlation coefficient, it is necessary to focus on the voltage across the diode (Vout). Fig. 14 shows a zoom of the evolution of this voltage when the transistor is switching on and off for different values of the switched current (from $1 \mathrm{~A}$ to $8 \mathrm{~A}$ ).

When the transistor is switching on, a voltage step $\Delta \mathrm{V}_{\text {out }}$ appears across the diode (Fig. 3) due to the decrease of the current across the equivalent stray inductance $L_{P}$. L $L_{P}$ corresponds to internal inductance of discrete components and to the stray inductance introduced by the PCB. The duration $(\Delta \mathrm{T})$ of the voltage step is linked to the reverse recovery current which is proportional to the switched current. The maximum time delay $\Delta \mathrm{t}$ introduced is about $30 \mathrm{~ns}$. After this first step, the most important $d V_{\text {oUT }} / d t$ appears. A resonance phenomenon between $L_{P}$ and stray capacitor of switches explains the oscillations that appear on the voltage. The amplitude of those oscillations is quite constant which is why $C\left[p_{U_{k}} ; \widehat{p_{U}}\right]$ is very close to 1 . On the contrary, $C\left[p_{D_{k}} ; \widehat{p_{D}}\right]$ is not always close to $1([0.69 ; 0.99])$ because when the transistor is switching off, the $d V_{\text {OUT }} / d t$ depends on the level of the switched current. The lower the current is (close to $1 \mathrm{~A}$ ), the less the pulses correlate $\left(C\left[p_{D_{k}} ; \widehat{p_{D}}\right] \approx 0.7\right)$. This is the case of pulses index 5 and 30 . On the contrary, the correlation coefficient is higher $(\approx 0.95)$ when the current is maximum (pulses index 18 and 43). The highest value of the correlation coefficient $(\approx 0.99)$ is obtained for middle values of current of about 3.7 A (pulses index 11, 24 and 35).
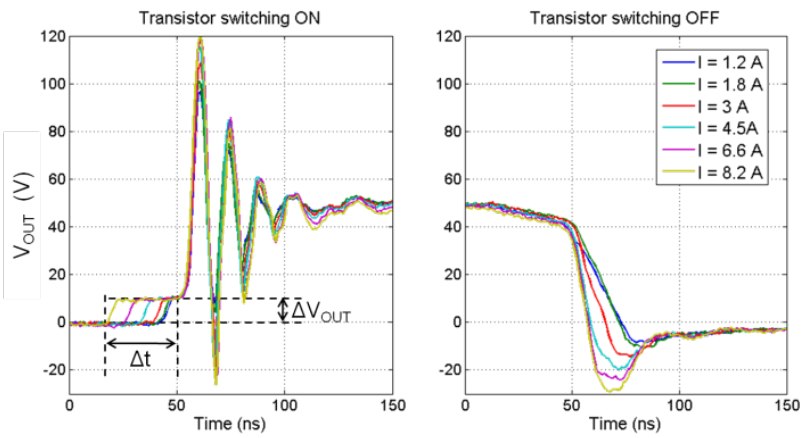

Fig. 14: Zoom on the voltage across the diode when the transistor is switching on and off.

In order to check the relevance of the correlation coefficient as a criteria to select representative pulses, the CM current calculated thanks to the pulses with the highest correlation coefficient ( $p_{U-38}$ and $\left.p_{D-35}\right)$ is compared in the frequency domain to the measured current (Fig. 15). The comparison reveals that the pulses extracted from the measurement are as efficient as the two equivalent ones in reconstructing the measured spectrum of $i_{C M}$.

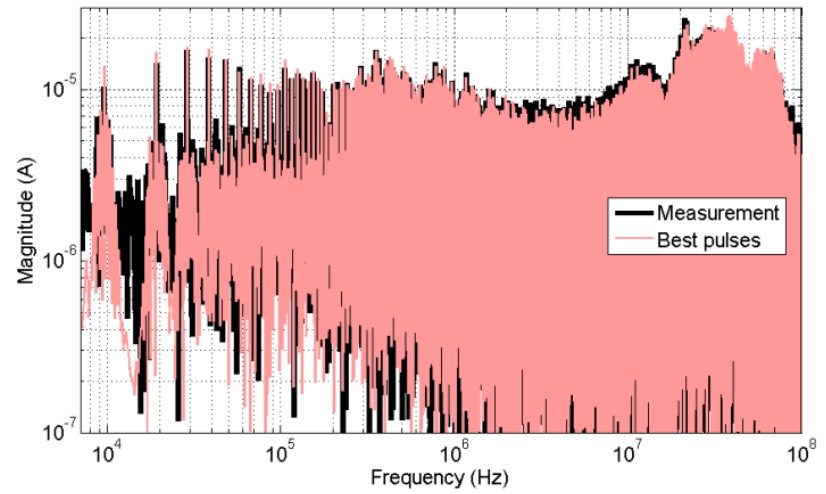

Fig. 15: Comparison of the spectrums either calculated with the measurement of the CM current or computed using the best pulses ( $p_{U-38}$ and $\left.p_{D-35}\right)$ according to the correlation with equivalent pulses.

2) Selection using the maximum of the mean of the correlation coefficient of each pulse

Another way to extract relevant pulses is to calculate the correlation coefficient between all the pulses. The matrixes $\left(\mathrm{C}_{\mathrm{U}}\right.$ and $\left.\mathrm{C}_{\mathrm{D}}\right)$ of all the correlation coefficients are represented in Fig. 16. The means of the correlation coefficient for each pulse $\left(\left\langle C_{U}\right\rangle_{k}\right.$ or $\left.\left\langle C_{D}\right\rangle_{k}\right)$ are calculated according to (8) and enable to quantify how each pulse is similar to all the others (it corresponds to the mean of the matrix rows). $\left\langle C_{U}\right\rangle_{k}$ and $\left.<C_{D}\right\rangle_{k}$ are represented in Fig. 17-A.

The highest values of $\left\langle C_{U}\right\rangle$ and $\left\langle C_{D}\right\rangle$ are obtained for the same pulses as previously ( $p_{U-38}$ and $p_{D-35}$ ). The lowest values are obtained for pulses where the switched current is low (around index 5 and 30) and where the switched current is high (around index 15 and 45) (Fig. 17-B). The reconstruction with pulses where the switched current takes extreme values is represented on Fig. 18. It appears that the spectrum of the real $\mathrm{CM}$ current is included between those two calculated currents. 

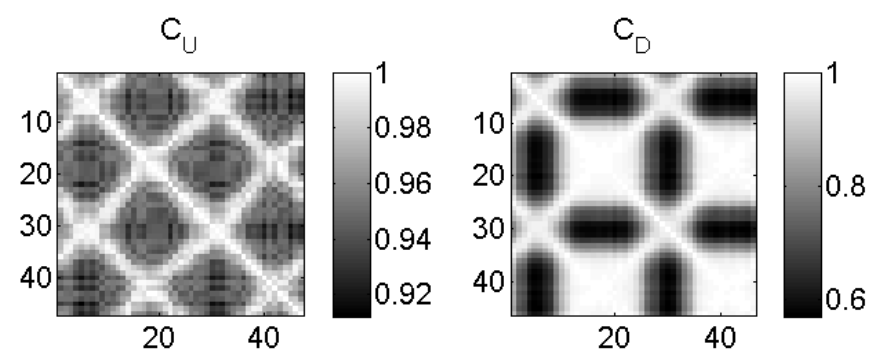

Fig. 16: Correlation matrix between all measured pulses.

$$
\begin{aligned}
& <C_{U}>_{k}=\frac{1}{n} \cdot \sum_{i=1}^{N_{U}} C\left[p_{U_{i}} ; p_{U_{k}}\right] ; \forall k \in\left[1, N_{U}\right] \\
& <C_{D}>_{k}=\frac{1}{n} \cdot \sum_{i=1}^{N_{D}} C\left[p_{D_{i}} ; p_{D_{k}}\right] ; \forall k \in\left[1, N_{D}\right]
\end{aligned}
$$

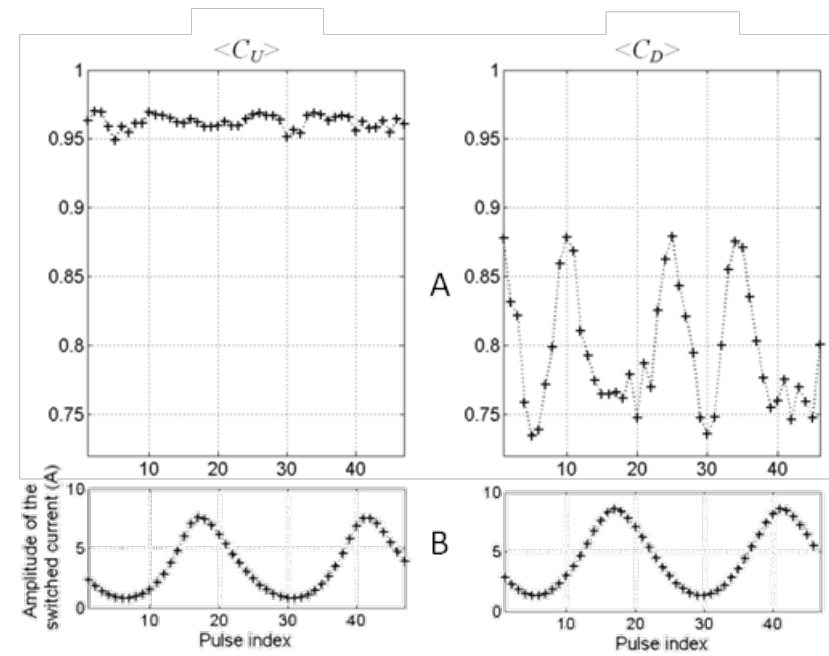

Fig. 17: A - Mean of correlation coefficient as a function of pulse index. B Magnitude of the switched current versus pulse index.

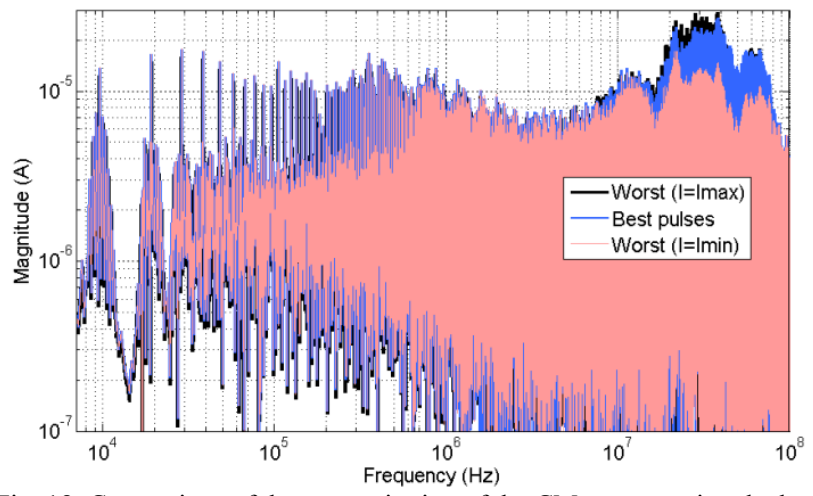

Fig. 18: Comparison of the reconstitution of the $\mathrm{CM}$ current using the best pulses and the worst ones when the switched current is maximum or minimum.

This part shows that it is possible to extract from the measurement two relevant pulses that enable to calculate the broad band spectrum of a CM current. Those pulses are extracted at a specific instant which remains to be determined. Moreover, the reconstruction of a $\mathrm{CM}$ current with pulses where the switched current takes extreme values allows to find the extreme limits of the real CM current.
In order to evaluate the robustness of the method (FRM), we propose in the next part to reconstitute a $\mathrm{CM}$ current from theoretical switching instants and the best extracted pulses.

\section{E. Reconstitution from theoretical switching instants.}

The converter is driven by an intersective (natural) PWM where a sinusoidal and a sawtooth signal are compared. The switching instants can easily be computed since they are defined by the intersection between those two signals. However, a variable time delay (noted $\Delta t$ in Fig. 14) appears between those instants and the real apparition of the pulses $(0 \mathrm{~ns}<\Delta \mathrm{t}<30 \mathrm{~ns})$ due to the reverse recovery current of the diode. To take into account this time delay, a maximum random time delay (with $\Delta \mathrm{t}_{\max }=30 \mathrm{~ns}$ ) is added to the calculated switching instants. The comparison between the measurement and the reconstitution with the two best pulses and the calculated switching instants with and without time delay are represented in Fig. 19. It appears that the calculated switching instants with an additional time delay allow to reconstitute the real $\mathrm{CM}$ current. However, the added time delay has a high influence in the highest frequencies and causes a diminution of the spectrum level. It can be explain by the spread spectrum phenomena [11-13]. Considering the high influence of the delay on the spectrum, simulations cannot afford not to take it into account in order to be accurate. It is remarkable that the FRM can easily include delays by addition of random time delay on switching instants.

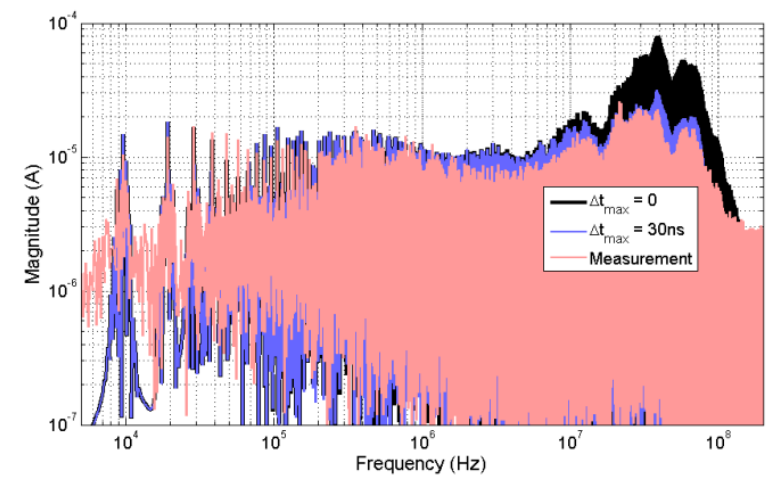

Fig. 19: Comparison between the measurement of the CM current and the calculation from the best pulses and the calculated switching instants without time delay and with a maximum random time delay of $30 \mathrm{~ns}$.

\section{Application to a Three Phase InVerter}

The following section deals with the application of the FRM to a three phase inverter connected to a synchronous motor. The experimental setup used is represented in Fig. 20. It is mainly composed by an inverter and a synchronous motor linked by one meter of unshielded cable. The wound rotor of the motor is fed by a linear continuous supply. The synchronous motor is mechanically coupled to a DC motor which constitutes a mechanical load. Electrical insulation between the two motors is insured. A DC power supply feeds the system and a common mode filter is inserted between the power supply and the inverter. A feedback torque control has been realized with a d-q transformation to drive the electrical machine and the fundamental of the power currents is chosen equal to $50 \mathrm{~Hz}$ (LF period of $20 \mathrm{~ms}$ ). The switching frequency of the inverter is fixed at $5 \mathrm{kHz}$. The $\mathrm{CM}$ current is measured 
at the inverter output using a current probe (Pearson 4109) which measures the sum of the currents in the three phases.

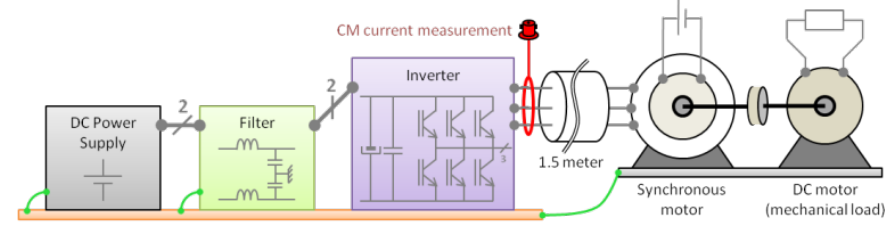

Fig. 20: Experimental setup used to study the CM current generated by a three phase inverter.

The CM current is acquired on a quarter of the LF period (5 $\mathrm{ms}$ ) and represented in Fig. 21. Because of the topological symmetry of the inverter, the pulses generated by a positive variation of the $\mathrm{CM}$ voltage of the inverter $\left(p_{U_{k}}\right)$ are considered as the opposite of the ones generated by a negative variation of the $\mathrm{CM}$ voltage. Consequently, only the $p_{U_{k}}$ pulses will be considered.

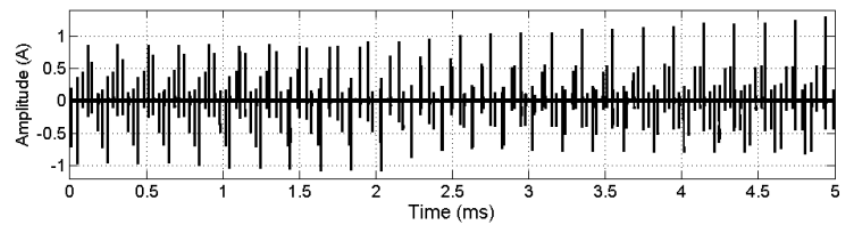

Fig. 21: Temporal acquisition on the quarter of a LF period of the CM current generated by the inverter.

\section{A. Pulse selection using the mean of the correlation coefficient}

All pulses are extracted from the measurement of one quarter of the LF period. As previously, the correlation coefficients between all the pulses are calculated and the correlation matrix is represented in Fig. 22-a. The mean of the correlation coefficient is then calculated (Fig. 22-b). In order to explain the difference between the pulses, the waveforms of four typical pulses are represented (Fig. 22-c). Pulses are similar to damped sines on which are superimposed at higher frequencies, oscillations with a variable magnitude. The mean of the correlation coefficient allows to extract the most relevant pulse $\left(\left\langle C_{U}\right\rangle=0.95\right)$ and the less relevant one $\left(\left\langle C_{U}\right\rangle\right.$ $=0.81$ ).
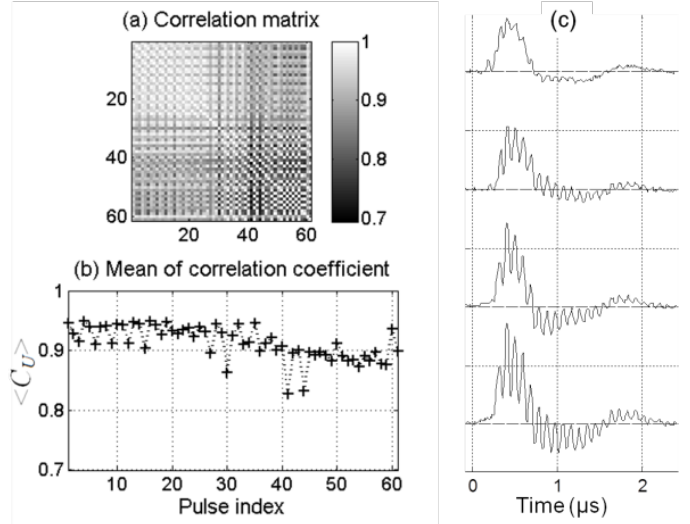

Fig. 22: (a) Correlation matrix representing the correlation coefficient between all the pulses - (b) Representation of $\left\langle C_{U}\right\rangle-$ (c) Waveform of four typical pulses contained in the quarter of the LF period of the CM current.

\section{B. Reconstitution from the most and the less relevant pulses} As previously, artificial currents containing only one selected pulse were reconstituted using the FRM. The comparison between the measurement of the $\mathrm{CM}$ current and the reconstitutions using the best and the worst pulses is represented in Fig. 23. The reconstituted CM using the best pulse shows a good similarity with the measurement from the switching frequency up to about $20 \mathrm{MHz}$. Beyond, the noise measurement does not allow to compare the results. Moreover, the reconstituted $\mathrm{CM}$ current using the worst pulse differs from measurement beyond about $1 \mathrm{MHz}$. In particular, the resonance that appears at $10 \mathrm{MHz}$ is more attenuated which could be expected since the oscillations that appears on the temporal waveform of this pulse are attenuated.

This study carried out on a three phase inverter shows that the FRM is also suitable for structures where the switching mechanisms are more complex than the ones involved in the PWM half bridge converter.

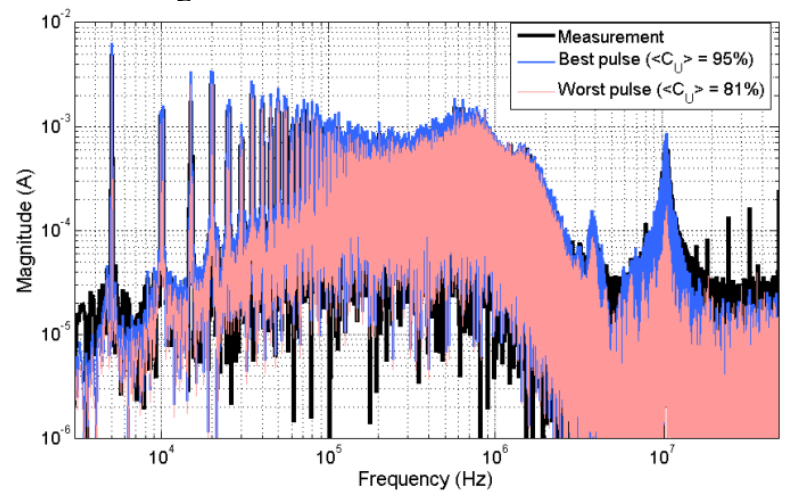

Fig. 23: Comparison of the measurement and the reconstitution of the $\mathrm{CM}$ current using the best and the worst pulses.

\section{OPERATION OF THE FRM IN A TIME DOMAIN SimUlation}

In order to quantify the gain of time brought by the FRM, the previous application (synchronous motor connected to an inverter) was implemented to Simplorer, a time domain solver. The simulation schematic is represented in Fig. 24 as well as the detail of the elements. The objective is not to precisely determine the level of disturbances generated by the application but to compare the duration needed to complete the simulation. A three phase inverter is implemented to Simplorer using basic models of MOS transistor (available in the library of the software) and the synchronous machine is represented by an equivalent circuit model.

In order to determine the spectrum of the $\mathrm{CM}$ current, a first simulation is completed during a LF period (Tend $=20 \mathrm{~ms}$ ) with a fix sampling period (Ts) of $10 \mathrm{~ns}$; which allows to calculate the spectrum up to $50 \mathrm{MHz}$. This classical simulation lasts about $2600 \mathrm{~s}$ (Table I).

In order to apply the FRM, two simulations are needed. The first one, which lasts about $245 \mathrm{~s}$, consists in running a fast simulation during a LF period with a sampling period of $100 \mathrm{~ns}$ to quickly calculate the control signals of the switches. The second one, which lasts only $14 \mathrm{~s}$, consists in precisely calculating the firsts $\mathrm{CM}$ pulses during $100 \mu$ s with a sampling period of $10 \mathrm{~ns}$. Those data are then exported to Matlab for post-treatment which lasts $11 \mathrm{~s}$. It consists in loading and interpolating data, calculating the switching instants from the 
control signals (by thresholding), extracting one pulse and convolving it to the switching instants in order to reconstitute the global CM current. The different steps of computation with the associated time durations are summarized on Table I. Finally, the global time needed to determine the CM current with the FRM is about $270 \mathrm{~s}$. The resulting spectrums of both simulations (Fig. 25) show that the FRM gives a quite similar result with an important gain of time. In our case, this method allows to divide the simulation time by about 10 .

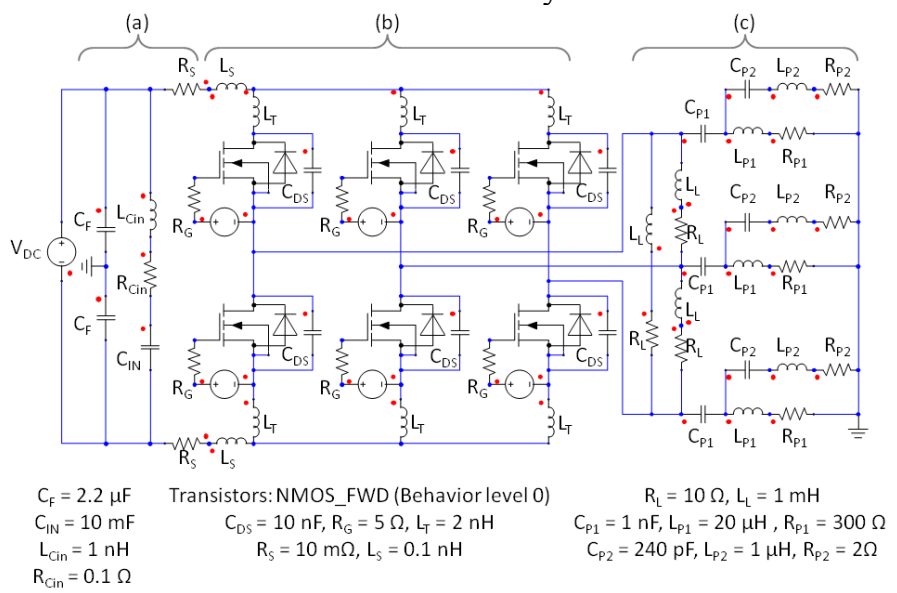

Fig. 24: Electrical diagram of the inverter implemented to Simplorer - (a) DC power supply and CM filter - (b) Inverter - (c) Synchronous Machine

\section{TABLE I}

Time Simulation ReQuired to CAlculate THE CM CURRENT.

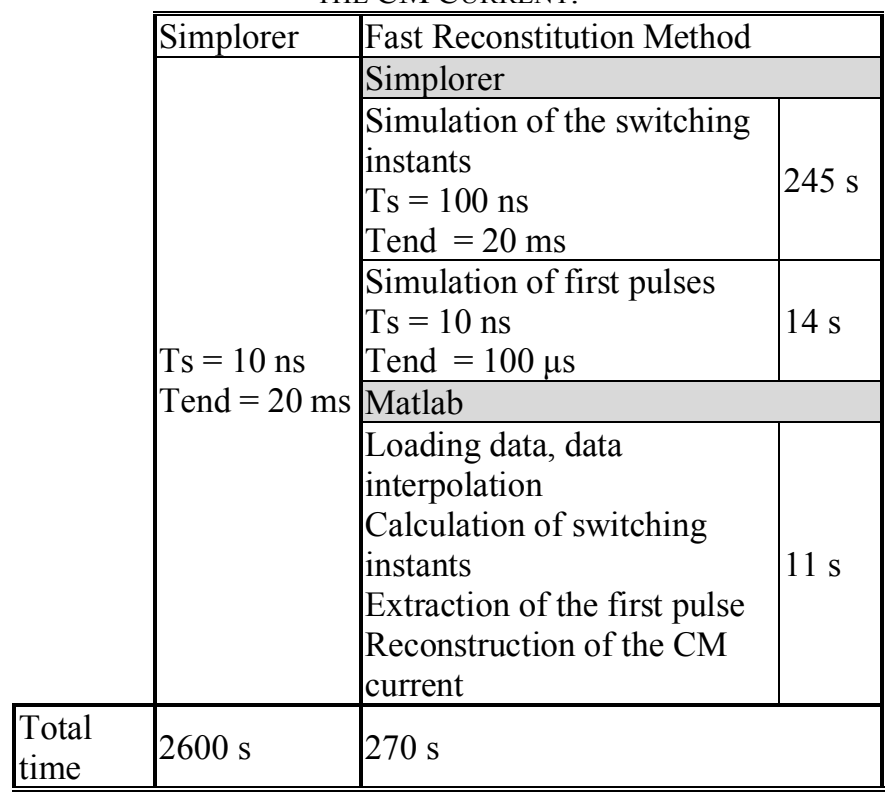

Sampling period and duration of the simulation are noted Ts and Tend respectively.

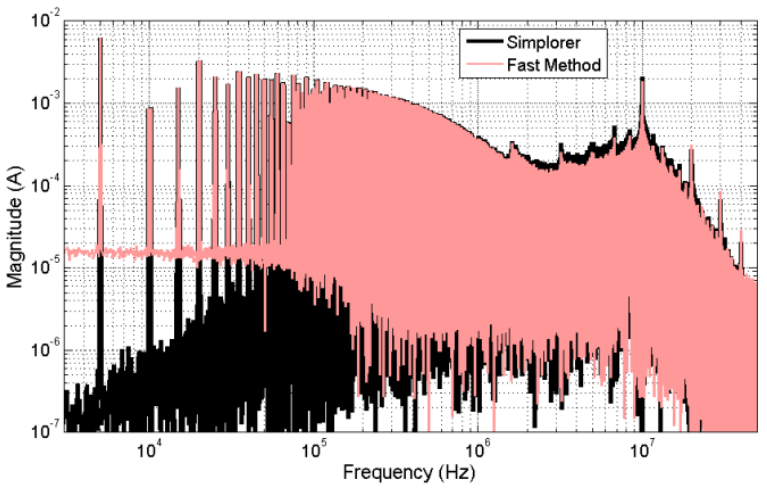

Fig. 25: Comparison in the frequency domain of the spectrums obtained directly with Simplorer and with the FRM.

\section{CONCLUSION}

This paper demonstrates that it is possible, knowing the switching instants, to reconstitute the CM current spectrum from the knowledge of only one precise CM pulse. This pulse can be extracted from measurement but also determined by simulation, which allows to considerably reduce the computation duration of the time domain simulation. A synoptic of this Fast Reconstitution Method (FRM) which resumes its principle, is represented in Fig. 2.

To make this method completely effective, the exact switching instant chosen to extract the most relevant pulse has to be determined. This study shows that this instant is linked to the magnitude of the switched current. This value must be compared to the extreme values of the switched current and to the technology and the voltage and current range of the switches.

\section{REFERENCES}

[1] M. Moreau, N. Idir, et P. Le Moigne, "Modeling of Conducted EMI in Adjustable Speed Drives," Electromagnetic Compatibility, IEEE Transactions on, vol. 51, $\mathrm{n}^{\circ}$. 3, p. 665-672, 2009.

[2] M. Beltramini et al., "Comparison of different inverter architectures and controls in terms of conducted EMI," in Industrial Technology (ICIT), 2010 IEEE International Conference on, p. 717-722, 2010.

[3] T. Wernicke, S. Dieckerhoff, T. Kirfe, G. Feix, S. Guttowski, et H. Reichl, "Experimental characterization methods for power MOSFET assemblies," in Power Electronics and Applications, 2007 European Conference on, p. 1-9, 2007.

[4] J. Schanen, C. Martin, D. Frey, et R. Pasterczyk, "Impedance criterion for power modules comparison," Power Electronics, IEEE Transactions on, vol. 21, $\mathrm{n}^{\circ} .1$, p. 18-26, 2006.

[5] C. Martin, J. Schanen, J. Guichon, et R. Pasterczyk, "Analysis of Electromagnetic Coupling and Current Distribution Inside a Power Module," Industry Applications, IEEE Transactions on, vol. 43, n . 4, p. 893-901, 2007.

[6] J. Ben Hadj Slama, S. Hrigua, F. Costa, B. Revol, et C. Gautier, "Relevant parameters of SPICE3 MOSFET model for EMC analysis," in Electromagnetic Compatibility, 2009. EMC 2009. IEEE International Symposium on, p. 319-323, 2009.

[7] R. Withanage, N. Shammas, S. Tennakoorr, C. Oates, et W. Crookes, "Igbt Parameter Extraction for the Hefner IGBT Model," in Universities Power Engineering Conference, 2006. UPEC '06. Proceedings of the 41st International, vol. 2, p. 613-617, 2006.

[8] Qian Liu, Fei Wang, et D. Boroyevich, "Modular-TerminalBehavioral (MTB) Model for Characterizing Switching Module Conducted EMI Generation in Converter Systems," Power Electronics, IEEE Transactions on, vol. 21, n . 6, p. 1804-1814, 2006.

[9] C. Jettanasen, F. Costa, et C. Vollaire, "Common-Mode Emissions Measurements and Simulation in Variable-Speed Drive Systems," Power Electronics, IEEE Transactions on, vol. 24, n. 11, p. 2456- 
2464, 2009.

[10] D. Labrousse, B. Revol, et F. Costa, "Common-Mode Modeling of the Association of $\mathrm{N}$-Switching Cells: Application to an ElectricVehicle-Drive System," Power Electronics, IEEE Transactions on, vol. 25, n. 11, p. 2852-2859, 2010.

[11] F. Mihalic et D. Kos, "Conductive EMI reduction in DC-DC converters by using the randomized PWM," in Industrial Electronics, 2005. ISIE 2005. Proceedings of the IEEE International Symposium on, vol. 2, p. 809-814 vol. 2, 2005.

[12] D. Gonzalez et al., "Conducted EMI Reduction in Power Converters by Means of Periodic Switching Frequency Modulation," Power Electronics, IEEE Transactions on, vol. 22, n. 6, p. 2271-2281, 2007.

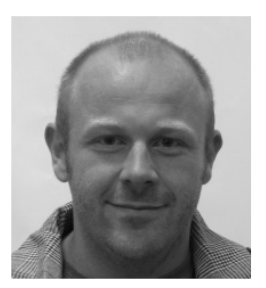

Denis Labrousse was born in France in 1981. He received the M.S. degree, in 2007 and the Ph.D. degree in electrical engineering from the Ecole Normale Superieure de Cachan (ENS de Cachan), Cachan, France, in 2010. His Ph. D. focused on electromagnetic compatibility of power electronics converters and was performed in the team Integrated Power Electronics and Materials (IPEM) in the laboratory Systèmes et Applications des Technologies de l'Information et de l'Energie (SATIE ENS de Cachan), Cachan, France. He currently continues his research in the field of power electronics.

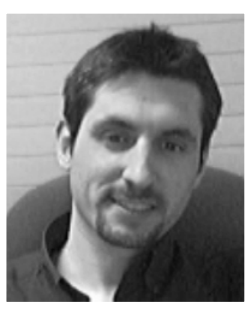

Bertrand Revol was born in France in 1976. He received the Engineer degree of ENSIEG from the Institut National Polytechnique de Grenoble, Grenoble, France, in 2000, and the Ph.D. degree in electrical engineering from the University Joseph Fourier, Grenoble, France, in 2003.

Since September 2004, he has been an Assistant Professor at the High Normal School of Cachan (ENSC), Cachan, France, where he carries out research in the laboratory of SATIE. These principal fields of research relate to the EMC of the power electronics converters.

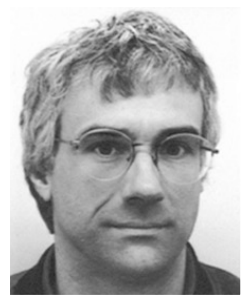

François Costa (M'95) received the Ph.D. degree in electrical engineering from the University of ParisSud, Orsay, France, in 1992. He has been with the Static Converter Group, Systèmes et Applications des Technologies de l'Information et de l'Energie (SATIE ENS de Cachan) Laboratory, Cachan, France, since 1989. He is also a Full Professor at the Institut Universitaire de Formation des Maîtres de Créteil, Créteil, France, where his teaching covers power electronics and EMI in static converters. Since 1999, he has been the Leader of the Power Electronics Team. His research concerns HF medium-power converters, EMI issues and modeling, HF instrumentation, integration in power electronics, and piezoelectric converters. 